DOE-ER40757-039

IASSNS-HEP 95/20

CPP-94-3

June, 1994

\title{
Quarks in the Skyrme-'t Hooft-Witten Model
}

\author{
L.C. Biedenharn \\ Center for Particle Physics, Department of Physics, University of Texas at Austin, \\ Austin, TX 78712 \\ L.P. Horwitz ${ }^{(a)}$ \\ School of Natural Sciences, Institute for Advanced Study \\ Princeton, NJ 08540
}

\begin{abstract}
The three-flavor Skyrme-'t Hooft-Witten model is interpreted in terms of a quark-like substructure, leading to a new model of explicitly confined color-free "quarks" reminiscent of Gell-Mann's original pre-color quarks, but with unexpected and significant differences.

PACS numbers: 12.38.Aw, 12.4.Aa, 21.60.Fw
\end{abstract}

The standard theory for strong interaction physics is, by consensus, QCD, but the inherent difficulties in applying this theory to long-range low-energy phenomena (meson-nuclear physics, say) have so far been insuperable. In the mid-seventies 't Hooft [四] demonstrated that the gauged color group $S U\left(N_{c}\right)$, with $N_{c} \rightarrow \infty$, provided an approximate approach to strong interactions. In this limit, mesons have masses that scale as $\left(N_{c}\right)^{0}$ and the meson resonances are narrow with widths that scale as $\left(N_{c}\right)^{-1}$. In contrast, baryons (which contain $N_{c}$ quarks) have masses that scale as $\left(N_{c}\right)^{1}$ with sizes and shapes that have an $N_{c}$ independent limit. Witten[2] recognized that, for baryons, these are characteristics of a soliton, reviving an earlier (topological) strong interaction model of Skyrme [3].

The Skyrme-'t Hooft-Witten (S'tW) model of baryons results in a classical soliton solution of the nonlinear chiral $S U\left(N_{f}\right) \times S U\left(N_{f}\right)$ model, from which the quantal baryonic states can be projected. The crucial new ingredient in the S'tW model, due to Witten, is the anomaly term. The implications of this anomaly term are truly remarkable, and this term accounts for the impressive qualitative (structural) agreements of the model with observation. For two-flavors the anomaly does not exist and the $\mathrm{S}$ 'tW model is topologically trivial, essentially equivalent, in fact, to the old strong-coupling spin-isospin model. Deeper insight into the structure of the twoflavor S'tW model came from the quark hedgehog (large- $N_{c}$ quark) analysis [4]. This analysis, combined with K-symmetry, as discussed by Mattis and Braaten[5], led to predictions for twoflavor meson-baryon scattering in surprisingly good agreement with experiment.

The situation for the three-flavor S'tW model - - as a direct consequence of the anomaly term - - is totally different. Here the analysis in terms of large- $N_{c}$ quarks is - - as we will prove - - 
incorrect in the literature. Part of the problem is the understandable, but unfortunate, confusion caused by denoting two distinct concepts by the same symbol. The S'tW model, when analyzed and interpreted in the way we propose, can be seen as a model based on quark-solitons, which are generalized (topological) "quarks" strongly reminiscent of Gell-Mann's original (pre-color) "mathematical" quarks [6] - - though not without some unusual features of their own, as we shall show.

The three-flavor S'tW model is defined[2] by the Poincaré invariant action:

$$
S=\int d^{4} x L+n \Gamma,(n \in Z),
$$

where $\Gamma$ is the anomaly and $L$ is the Skyrme Lagrangian,

$$
L=\frac{F_{\pi}^{2}}{16} \operatorname{Tr}\left\{\left[\partial_{\mu} U \partial^{\mu} U\right]\right\}-\frac{1}{32 e^{2}} \operatorname{Tr}\left\{\left[\left(\partial_{\mu} U\right) U,\left(\partial_{v} U\right) U\right]^{2}\right\}
$$

with $U\left(x_{\mu}\right) \in S U(3), F_{\pi} \cong 186 M e v$, and $e$ is a dimensionless constant. The anomaly[2] cannot be written as an integral over space-time, but appears in the form:

$$
\Gamma=\frac{1}{240 \pi^{2}} \int d \Sigma^{i j k l m} \operatorname{Tr}\left(V_{i} V_{j} V_{k} V_{l} V_{m}\right)
$$

with $V_{j} \equiv-U^{-1} \partial_{j} U, U \in S U(3)$, and $d \Sigma^{i j k l m}$ a volume element in an extended five-dimensional space; the boundary of the integration region is compactified space-time.

Topological considerations enter as follows: for a given time $t$, the matrix $U(t, x)$ is a mapping from $\boldsymbol{R}^{3}$ into $S U(3)$. The proper boundary conditions add the point at infinity to three-space compactifying it to $S^{3}$. As is familiar from Witten's work, [2] the equivalence classes of all such maps are classified by the homotopy group $\pi_{3}(S U(3))=Z$, (the integral baryon number $\mathrm{B}$ ).

For three-flavors the lowest energy $B=1$ soliton of the $\mathrm{S}^{\prime} \mathrm{tW}$ model is the $3 \times 3$ matrix:

$$
\Sigma(r)=\left(\begin{array}{cc}
\exp \left(\frac{2 i}{F_{\pi}} \tau \cdot \hat{r} F(r)\right) & 0 \\
0 & 1
\end{array}\right),
$$

where $\tau$ denotes the $2 \times 2$ isospin matrices and $F(r)$ is determined from the Euler-Lagrange equations. The soliton $\Sigma$ has the symmetry $S U(3)_{\text {flavor }} \times S U(2)_{\text {spin }}$, where $S U(3)_{\text {flavor }}$ is realized by the adjoint action and $S U(2)_{\text {spin }}$ by transformations on $\mathbf{r}$ (generators $\mathbf{J}$. Eq.(4) shows a special "K-symmetry" in that $\Sigma$ is invariant under combined isospin-spin rotations: $(\mathbf{I}+\mathbf{J})(\Sigma)=0$, and, moreover, the $S U(3)_{\text {flavor }}$ hypercharge generator $Y$ also leaves $\Sigma$ invariant: $[Y, \Sigma]=0$.

The quantal eigenstates of the S'tW model are projected from the soliton $\Sigma$, and are monopolar harmonics [7], sections of a fiber bundle over the coset manifold $S U 3 / U 1$. These monopolar harmonics are specializations of the $S U 3$ rotation matrices over eight angles $\left(\alpha_{1}, i=1 \ldots 8\right)$, with the angle $a_{8}$ determined by the sectional map. The $S U(3) \times S U(3)$ symmetry of the $S U(3)$ rotation matrices (generated by left and right actions on the $S U(3)$ manifold) reduces to the symmetry $S U(3)_{\text {flavor }} \times S U(2)_{\text {spin }}$ for the monopolar harmonics, with the anomalous right action hypercharge $Y_{R} \rightarrow N_{c} B / 3$. 
Can one construct a large- $N_{c}$ quark model unitarily equivalent to this S'tW three- flavor model? Manohar 4 was the first to attempt this and his model was based on the hedgehog quark:

$$
\left|\Sigma_{q}\right\rangle \equiv(|u\rangle \otimes|\downarrow\rangle-|d\rangle \otimes|\uparrow\rangle)
$$

where (as in the two-flavor case) $\boldsymbol{K}\left|\Sigma_{q}\right\rangle=0$, where $\boldsymbol{K}=\boldsymbol{I}+\boldsymbol{J}$. What distinguishes this ket vector, $\left|\Sigma_{q}\right\rangle$, from the two-flavor case is the set of allowed operations: for the three-flavor case, one allows arbitrary $S U(3)_{\text {flavor }}$ and $S U(2)_{\text {spin }}$ transformations to act on $\left|\Sigma_{q}\right\rangle$. Transforming $\left|\Sigma_{q}\right\rangle$ by $R(g)$, where $g \in S U(3)_{\text {flavor }}$, carries $\left|\Sigma_{q}\right\rangle$ to sufficiently many independent states to enable one to determine the six basis states of the defining irrep of $S U(3)_{\text {flavor }} \times S U(2)_{\text {spin }}$.

Consider now the anti-symmetrized color singlet $N_{c}$-hedgehog quark state:

$$
\left|\Sigma_{q}\right\rangle_{N_{c}} \equiv \underbrace{\left(\left|\Sigma_{q}\right\rangle \otimes\left|\Sigma_{q}\right\rangle \ldots \otimes\left|\Sigma_{q}\right\rangle\right)}_{N_{c} \text { times }} \chi_{\text {color singlet }} \text {, }
$$

If we perform the transformation: $\left|\Sigma_{q}\right\rangle \rightarrow R(g)\left|\Sigma_{q}\right\rangle, g \in S U(3)_{\text {flavor }}$ this induces a general transformation on $\left|\Sigma_{q}\right\rangle_{N_{c}}$. The states spanned by $\left\{R(g)\left|\Sigma_{q}\right\rangle_{N_{c}}, g \in S U(3)\right\}$ are precisely the states of the totally symmetric irrep $\left[N_{c} \dot{0}\right]$ in $S U(6)$. The $S U(3) \times S U(2)$ structure of the irrep $\left[N_{c} \dot{0}\right]$ in $S U(6)$ is well known 8$]$ : Every irrep $\left[m_{13} m_{23} 0\right] \times\left[m_{13} m_{23}\right]$ in $S U(3) \times S U(2)$ occurs once and only once, subject to lexicality and the constraint that $m_{13}+m_{23}=N_{c}$.

To compare the baryonic states in this large $N_{c}$ quark model with the baryonic states of the three-flavor S'tW model we see that we must choose $N_{c}=3 k$, with $k$ an integer. This comparison shows that there are serious discrepancies. In fact only for $N_{c}=3$ are the states the same. One finds that: (a) the spins in Manohar's model for the baryon tower are not all half-integral. (b) the multiplicity of the $S U(3)_{\text {flavor }}$ multiplets in the two systems is not the same, and (c) unlike the two-flavor hedgehog quark model, the multiplets for a given $N_{c}(=3 k)$ do not contain the multiplets for lower $N_{c}(=3(k-1))$. This means that the physically important lowest multiplets in the tower will be obtained only with $N_{c}=3$, which prevents using the large- $N_{c}$ limit.

To resolve these discrepancies, we remark that the proper three-flavor hedgehog quark is not defined by (6) but by the local [100] monopolar harmonic, which does not exist globally, since such an object is forbidden topologically. (More precisely these objects exist locally as confined triples). Let us assume that this [100] state does exist locally; what would it look like? Written as a matrix it would appear trivial, simply the $3 \times 3$ unit matrix. But recall that $S U(3)$ rotational wave functions realize the symmetry $S U(3)_{\text {left }} \times S U(3)_{\text {right }}$ in which (conventionally) the left generators obey time-reversed commutation rules. [9] Re-writing the $3 \times 3$ unit matrix to accord with this basis, we find that

$$
\left|\Sigma_{0}\right\rangle=(|u\rangle \otimes|\downarrow\rangle-|d\rangle \otimes|\uparrow\rangle+|s\rangle \otimes|\rightarrow\rangle)
$$

where $|\rightarrow\rangle$ denotes a new "sidewise", or spin 0, component. Clearly the state $\left|\Sigma_{0}\right\rangle$ is invariant under an octet of $S U(3) \boldsymbol{K}$-symmetry generators.

We must justify this radical step impled by (7), but before we do so let us remark that all of the difficulties noted above are resolved by this change. 
At first glance the introduction of a scalar $(S=0)$ "quark" is absurd; there is no experimental evidence for such an object. But before we dismiss this idea (which was, after all, abstracted from the S'tW model) let us be more careful and examine the right hypercharge (the baryon number in the model). We find $B=-2 / 3$. Thus the new $S=0, Y_{R}=B=-\frac{2}{3}$ state $i s$ an anti di-quark. There is credible evidence that the di-quark [6] exists. [10]

We conclude that the adjunction of the anti-diquark to define $\left|\Sigma_{0}\right\rangle$ is not completely unreasonable.

The resulting large- $N_{c}$ quark model is now straight-forward. We must use $N_{c}=3 k$ to agree with the states of the three-flavor S'tW model. The single "quark" basis implied by $\left|\Sigma_{0}\right\rangle$ consists of the nine states in $S U(3)_{\text {flavor }} \times S U(3)_{\text {right }}$. Thus a baryonic structure composed of $N_{c}$ such quarks consists of $\operatorname{dim}\left[N_{c} \dot{0}\right]_{U 9}$ symmetric states in $S U(9)$, since the color state is an anti-symmetric singlet in $S U\left(N_{c}\right)$. It is important to realize that this $N_{c}$-quark system consists of "baryons" with various baryonic charges (right hypercharge $Y_{R}$ ) since the original "quark" basis itself had two distinct baryonic charges $\left(\frac{1}{3}\right.$ and $\left.-\frac{2}{3}\right)$. Thus we must project from this system of $N_{c}$ "quarks" the states of $Y_{R}=B=1$ to accord with the $B=1$ states of the three-flavor $S$ ' $t W$ model. It is easily demonstrated that this projected $S U(3)_{\text {flavor }} \times S U(3)_{\text {right }} \times S U\left(N_{c}\right)$ "quark" model for $N_{c} \rightarrow \infty$ is unitarily equivalent to the three-flavor S'tW model since the spectrum of states is identical. Accordingly we propose to take this new "quark" model more seriously and examine its implications.

The results we have presented so far agree nicely with the S'tW model, and are, group theoretically, unassailable. Despite this, a more critical analysis shows there is a problem: following convention, we have used the symbol $N_{c}$ ambiguously, in two distinct, conflicting, ways. If the anomaly were absent, there would be no ambiguity: one simply takes $S U(3)_{\text {flavor }} \times S U(2)_{\text {spin }} \times$ $S U\left(N_{c}\right) Q C D$ in the 't Hooft limit $N_{c} \rightarrow \infty$ obtaining $S U(3)_{\text {flavor }} \times S U(2)_{\text {spin }}$ symmetry, defined ( a la Witten) on the manifold $S U(3) / U 1$. For clarity, let us henceforth call 't Hooft's parameter $N^{\prime} t$. Now consider the anomaly. First of all the anomaly cannot even be defined until we have constructed the limit $N_{\prime} t \rightarrow \infty$, obtaining a smooth manifold (over $N^{\prime} t$ ). Thus the anomaly, which is quantized, introduces a new integral parameter, confusingly called $N_{c}$ again. To avoid confusion let us call this Witten's parameter[11] denoted by $N_{W}$.

The limit $N_{\prime} \rightarrow \infty$ clearly eliminates the parameter $N^{\prime} t$ from the $S^{\prime} t W$ model, which means that in the $S^{\prime} t W$ model color has been totally confined and disappears from the model. The anomaly makes a profound difference. Witten's parameter $N_{W}$ must be 3 , which implies that right hypercharge measures the baryon quantum number B. On purely topological grounds, the anomaly forbids the existence of $S^{\prime} t W$ states with fractional $B$ (that is, non-zero triality[19]). Triples of objects, with composite triality zero, are, however, not forbidden if confined within the volume of the Skyrmion (or to even smaller distances). Thus even though color has disappeared we still have a remnant: quark confinement in triples. (This beautifully illustrates Gell-Mann's intuition that quarks may be fictitious (unobservable) in his classic pre-color paper. [6])

For consistency with both the large- $N_{t_{t}}$ quark model and the admissible quark triples, one must take the $N_{t} \rightarrow \infty$ limit to run over the integers $N^{\prime} t \equiv 0 \bmod 3$. Thus we see the true relationship [13] between 't Hooft's parameter $N^{\prime} t$ in the large- $N_{\prime^{\prime}}$ quark model and Witten's anomaly parameter $N_{W}$ is that $N^{\prime} t \equiv t \bmod N_{W}$ with $N_{W}=3$. (Here $t=0, \pm 1$ is the triality of 
the $S U(3)_{\text {flavor }}$ irrep.)

Accordingly we abstract from the S'tW model the following structure: a quark soliton is a confined 'solution' to the S'tW model having $B=\frac{1}{3}$, belonging to the broken spectrum generating symmetry $U(9) \subset S U(3)_{\text {flavor }} \times S U(3)_{\text {right }} \subset S U(3)_{\text {flavor }} \times S U(2)_{\text {spin }} \times U 1_{Y_{R}}$. Projected onto quantal states, this $B=\frac{1}{3}$ system has an infinite tower of states: $[100] \frac{1}{2},[220] \frac{1}{2},[310] \frac{1}{2},[310] \frac{3}{2},[400] \frac{3}{2} ; \ldots$ A finite subset of these soliton quark states can be generated by $N^{\prime} t \equiv 1 \bmod 3$ confined colorless totally symmetrized light (mass $\sim N_{t}^{-1}$ ) "quarks", comprising the states: $[100] \frac{1}{2} \ldots,\left[N_{\prime_{t}} 00\right]\left(\frac{2 N_{t_{t}}+1}{6}\right)$. The smallest such subset $N_{\prime}=3$ ) are the six $B=1 / 3$ pre-color Gell-Mann quarks: [100]1/2, and, in addition, the three (structurally essential) $B=-\frac{2}{3}$ "quarks" [100]0, that is, nine $[100]_{\text {flavor }} \times[100]_{\text {right }}$ states in all. Composite projected states of three quark solitons are baryons $(\mathrm{B}=1)$.

Quark soliton composites are automatically equipped with a certain form of interaction: it is easily shown 14] that in the limit $N_{\prime}^{\prime}=\infty$ all interactions are local in the group (coset) manifold coordinates. This local interaction predicts three-flavor meson-baryon scattering 15 for $N^{\prime} t=\infty$, generalizing the earlier (two-flavor) results of Mattis-Braaten.[5]

The manifold coordinates (seven dimensional) are dual to the representation-label space (seven dimensional) so that finite $N^{\prime} t$ is to be associated with non-locality which can be modelled by group-theoretically defined tensor operators. Expressed differently the loss of color degrees of freedom by explicit confinement is compensated by the freedom to model, group-theoretically, interactions in composite (large- $N_{\prime} t$ ) states. The model we propose implies structurally many features previously introduced heuristically. Among these are: suppression of orbital angular momentum (quark-diquark baryonic excited states), explicit ('bag model') quark confinement, massless quarks, zero flavor triality, the 'spin-baryonic charge' rule $(2 S \equiv B \bmod 2)$ and suppression of nuclear 'hidden-color' effects.

The $S U(3)_{\text {color }}$ symmetry of QCD is a deep and profound organizing principle in particle physics. It will be interesting to see if soliton quarks, which define a color-free approach to long-range, low energy QCD structures, can successfully approximate the real meson-nuclear world.

\section{Acknowledgements:}

We would like to thank Dr. Michael Mattis (LANL) for interesting us in large- $N_{c}$ models and for many clarifying discussions. One of us (LPH) wishes to thank S.L. Adler for his hospitality at the IAS and the Monel Foundation for partial support. This work was supported in part by grant DE-FG03-93ER40757.

(a) Permanent address: School of Physics, Raymond and Beverly Sackler Faculty of Exact Sciences, Tel Aviv University, Ramat Aviv, Israel.

\section{References}

[1] G. 't Hooft, Nucl. Phys. B 72, 461 (1974); ibid. B 75, 461 (1974). 
[2] E. Witten, Nucl. Phys. B 223, 422 (1983); ibid. 223, 433 (1983).

[3] T.H.R. Skyrme, Proc. Roy. Soc. London A 260, 127 (1961), and Nucl. Phys. 31, 556 (1962).

[4] A.V. Manohar, Nucl. Phys. B 248, 19 (1984).

[5] M.P. Mattis and E. Braaten, Phys. Rev. D 39, 2737 (1989).

[6] M. Gell-Mann, Phys. Lett. 8, 214 (1964).

[7] L.C. Biedenharn and Yossef Dothan, "Monopolar Harmonics in $S U(3)_{f}$ as Eigenstates of the Skyrme-Witten Model for Baryons", From SU(3) to Gravity (Ne'eman Festschrift) (Cambridge University Press, Cambridge, England) 19 (1986).

[8] L.C. Biedenharn, A. Giovannini, and J.D. Louck, Math. Phys. 8, 691 (1967).

[9] L.C. Biedenharn and J.D. Louck, "Angular Momentum in Quantum Physics:" Cambridge University Press, (Cambridge, U.K.) (1981).

[10] M. Anselmino, E. Predazzi, S. Ekelin, S. Fredriksson, and D.B. Lichtenberg, Rev.Mod.Phys. 65, 1199 (1993).

[11] Witten had good reason to call this new parameter "the number of colors". He showed that the anomaly implied an electromagnetic interaction which, to agree with experiment, forced the anomaly parameter $N_{W}$ to be 3 , the physical number of colors. But we insist that Witten's anomaly parameter, $N_{W}$, is logically distinct from the parameter $\left(N^{\prime} t=\infty\right)$ used in defining the $S U(3) / U 1$ manifold itself.

[12] L.C. Biedenharn and E.C. Fowler, Nuovo Cim. 33, 1329 (1964).

[13] Note the amusing point: Gell-Mann chose the label "color" by analogy to physical color, which has a basis of three colors. Gell-Mann's analogy is even more apt in our case, where $N^{\prime} t \rightarrow \infty$ corresponds to the infinite number of colors (distinct wave-lengths) while the number of basis colors being three, corresponds to the modulus $N_{W}$.

[14] Using the same procedure for three-flavors as used by Manohar [4 and by Mattis-Braaten [5] for two flavors.

[15] L.C. Biedenharn and L.P. Horwitz, "On the Equivalence of the Skyrme-Witten and Large- $N_{c}$ Quark Models", to be published in Foundations of Physics. 